\title{
R-Matrix study of electron impact excitation and dissociation of $\mathrm{CH}^{+}$ions
}

\author{
K. Chakrabarti* , A. Dora ${ }^{\sharp}$, R. Ghosh ${ }^{\ddagger}$, B. S. Choudhury ${ }^{\ddagger}$ and \\ Jonathan Tennyson ${ }^{\dagger}$ \\ * Department of Mathematics, Scottish Church College, 1 \& 3 Urquhart Sq., Kolkata \\ 700006, India \\ \# Department of Chemistry, North Orissa University, Baripada 757003, Odisha, India \\ ¥ Department of Mathematics, Indian Institute of Engineering Science and \\ Technology, Shibpur, Howrah 711103, India \\ $\dagger$ Department of Physics and Astronomy, University College London, Gower St., \\ London WC1E 6BT, UK \\ E-mail: j.tennyson@ucl.ac.uk
}

\begin{abstract}
Electron impact excitation and electron impact dissociation of $\mathrm{CH}^{+}$ions are studied in the framework of the $R$-matrix method using the diatomic version of the UK molecular $R$-matrix codes. A configuration-interaction calculation is first performed to yield the potential energy curves of the lowest eight singlet and triplet states of $\mathrm{CH}^{+}$. Scattering calculations are then performed to yield vibrationallyresolved electronic excitations to the lowest three bound states, namely the a ${ }^{3} \Pi$, A ${ }^{1} \Pi$ and the $b^{3} \Sigma^{-}$. Electron impact dissociation cross sections are obtained from the assumption that all electronic excitations above the dissociation threshold result in dissociation. Bound states of $\mathrm{CH}$ and resonance positions and widths of Feshbach resonances in the e- $\mathrm{CH}^{+}$system are also calculated at the $\mathrm{CH}^{+}$equilibrium bond length $2.137 \mathrm{a}_{0}$.
\end{abstract}

\section{Introduction}

Molecular ions are a constituent of many low temperature plasmas and diffuse interstellar clouds, where collision of these ions with electrons play an important role governing their chemistry. Hydrocarbon ions, and in particular $\mathrm{CH}^{+}$, are found in the edge plasmas in those fusion reactors operating with graphite as plasma facing material (Tawara 1995). $\mathrm{CH}^{+}$ions also occur in the interstellar medium (ISM) where they were first detected and studied by Douglas \& Herzberg (1941) in interstellar molecular clouds (see also Douglas \& Herzberg (1942)).

Electron collision with $\mathrm{CH}^{+}$ions can lead to electronic excitation,

$$
e^{-}+\mathrm{CH}^{+} \longrightarrow \mathrm{CH}^{+*}+e^{-}
$$


where $\mathrm{CH}^{+*}$ refers to an excited state of the ion, or destruction of the ion either by dissociation,

$$
\begin{aligned}
e^{-}+\mathrm{CH}^{+} & \longrightarrow \mathrm{C}+\mathrm{H}^{+}+e^{-} \\
& \longrightarrow \mathrm{C}^{+}+\mathrm{H}+e^{-}
\end{aligned}
$$

or by dissociative recombination via an intermediate neutral resonant state $\mathrm{CH}^{* *}$,

$$
e^{-}+\mathrm{CH}^{+} \longrightarrow \mathrm{CH}^{* *} \longrightarrow \mathrm{C}+\mathrm{H} \text {. }
$$

Collision cross sections for these processes are therefore important for modeling of the plasma environment and to understand the chemistry of formation and destruction of $\mathrm{CH}^{+}$in the ISM (Godard et al 2012, Nagy et al 2013). There has been a long-running issue that the observed ISM concentration of $\mathrm{CH}^{+}$is persistently larger than that predicted by models (Hayden Smith et al 1973), see also Godard \& Cernicharo (2013), Myers et al (2015) and references therein. This issue has recently been considered at length by Faure et al (2017) who showed that the proper treatment of non-local thermodynamic equilibrium effects are essential to model the production of $\mathrm{CH}^{+}$ions in the ISM.

Several earlier works on the $\mathrm{CH}^{+}$states have been undertaken of which we report only a few. Lorquet et al (1971) calculated the potential energy curves of $\mathrm{CH}^{+}$and studied its metastable decomposition and predissociation. Molecular constants including Franck-Condon factors for $\mathrm{A}^{1} \Pi-\mathrm{X}^{1} \Sigma^{+}$transitions were calculated by Liszt \& Hayden Smith (1972) and Hakalla et al (2006). Tennyson (1988) reported the lowest three bound states of $\mathrm{CH}^{+}$while obtaining the bound states of $\mathrm{CH}$ and resonances in the $e^{-} \mathrm{CH}^{+}$system. Kanzler et al (1991) obtained the lowest seven $\mathrm{CH}^{+}$states of singlet and triplet symmetry. They also gave the dipole moments, transition moments, oscillator strengths and radiative lifetimes computed by quasi-degenerate many body perturbation theory. Better calculations were performed by Kowalski \& Piecuch (2001) and Barinvos \& van Hemert (2004) though both were restricted to the singlet states only. Sauer \& S̆pirko (2013) obtained potential energy curves of the ground and several excited states of $\mathrm{CH}^{+}$which reproduced available spectroscopic data with high accuracy. More recently, a comprehensive set of $\mathrm{CH}^{+}$curves of singlet, triplet and quintet symmetries were obtained by Biglari et al (2014) using the multi-reference conguration interaction (MRCI) method with large basis sets. They also obtained transition dipole moments which were then used to calculate average lifetimes of excited state vibrational levels. Empirical potential energy curves for the $\mathrm{X}^{1} \Sigma^{+}$and $\mathrm{A}^{1} \Pi$ states were obtained by Cho \& Le Roy (2016) using an analysis of all available spectroscopic and photodissociation data; nuclear motion calculations using these curves reproduced all data within their range of uncertainties.

On the collisional aspects, photodissociation studies on $\mathrm{CH}^{+}$were reported by Barinvos \& van Hemert (2004) and Boukaline et al (2005). Electron impact rotational excitation and de-excitation was reported by Lim et al (1999) using the $R$-matrix method. A second $R$-matrix calculation for rotational excitation of $\mathrm{CH}^{+}$, together 
with $\mathrm{HeH}^{+}$and $\mathrm{ArH}^{+}$was reported by Hamilton et al (2016) where they claimed an improvement of the rate coefficients for $\mathrm{CH}^{+}$over those of Lim et al (1999).

Some theoretical and experimental studies on the dissociative recombination and of rotational excitation of $\mathrm{CH}^{+}$also exist. A Multi Channel Quantum Defect Theory (MQDT) study of dissociative recombination of $\mathrm{CH}^{+}$and its isotopologue $\mathrm{CD}^{+}$was reported by Carata et al (2000) which were not in very good quantitative agreement with an earlier experimental study reported by Amitay et al (1996). Bannister et al (2003) reported an experimental study of the electron impact dissociation of $\mathrm{CH}^{+}$to the asymptotic states $\mathrm{C}^{+}\left({ }^{2} P\right)+\mathrm{H}\left({ }^{2} S\right)$. They measured absolute cross sections for electron impact dissociation of $\mathrm{CH}^{+}$ions producing $\mathrm{C}^{+}$ions. However, as discussed below, the initial distribution of $\mathrm{CH}^{+}$ions in these experiments, in which neither the vibrational nor electronic states of the ions involved were well-characterised, makes these results hard to use in plasma models.

In this article, we present electron impact excitation cross sections of $\mathrm{CH}^{+}$to some of its low-lying states and cross sections for electron impact dissociation to the lowest $\mathrm{C}^{+}\left({ }^{2} P\right)+\mathrm{H}\left({ }^{2} S\right)$ and $\mathrm{C}\left({ }^{3} P\right)+\mathrm{H}^{+}$asymptotes. To the best of our knowledge, there exist no previous $a b$ initio theoretical study on these aspects.

\section{Calculations}

\subsection{Method}

Our calculations are done using the $R$-matrix method, see reviews by Burke (2011) and Tennyson (2010). The starting point of such a calculation is the division of the configuration space into an inner region, here a sphere of radius $11 \mathrm{a}_{0}$, centred at the molecular centre-of-mass, which encloses the $N$-electron target $\mathrm{CH}^{+}$ion.

In the inner region, the wave function of the $(N+1)$-electron system $\left(\mathrm{CH}^{+}+e^{-}\right)$ is written as a close coupling (CC) expansion,

$$
\Psi_{k}=\mathcal{A} \sum_{i, j} a_{i, j, k} \Phi_{i}(1, \ldots, N) F_{i, j}(N+1)+\sum_{i} b_{i, k} \chi_{i}(1, \ldots, N+1),
$$

where $\mathcal{A}$ is the antisymmetrisation operator, $\Phi_{i}$ is the $n$-electron wave function of the $i^{\text {th }}$ target state, $F_{i, j}$ are continuum orbitals and $\chi_{i}$ are two-centre $L^{2}$ functions constructed by making all $(N+1)$-electrons occupy the target molecular orbitals (MOs), and takes into account the polarization of the $N$-electron target wave function in presence of the projectile electron.

Once a suitable target model is fixed, several scattering models can be constructed by choosing different expansions for the target wave function $\Phi_{i}$. Simple approximations, for example, static exchange (SE) and static exchange with polarization (SEP) models use only the target ground state represented by a Hartree-Fock (HF) self consistent field (SCF) wave function. Using the SE model, one can represent only shape resonances, while Feshbach resonances can be described with the SEP models, though these are not well represented without the inclusion of their parent electronic states. More 
sophisticated models, like the CC model used here, can include several target states and therefore are very suitable for representing Feshbach resonances and calculating electron impact cross sections.

The inner region wave function is used to build an $R$-matrix at the boundary of the $R$-matrix sphere and the $R$-matrix is then propagated to asymptotic distances and matched with known asymptotic functions (Noble \& Nesbet 1984). The matching yields the $K$-matrix from which all scattering observables can be extracted.

For diatomic targets Slater type orbitals (STOs) are known to provide a better target representation. Hence the diatomic version of the UK molecular $R$-matrix codes (Morgan et al 1998) which uses STOs were used. The continuum (Tennyson \& Morgan 1999) was represented by numerical orbitals in a partial wave expansion about the molecular centre of mass. These numerical orbitals were obtained as a solution of the radial Schrödinger equation with an isotropic Coulomb potential. A Buttle correction (Buttle 1967) was also used to allow for the arbitrary fixed boundary conditions imposed on the continuum basis orbitals.

\section{2. $\mathrm{CH}^{+}$target calcultions}

We have used the STOs of Cade \& Huo (1967) which consisted of $5 s$-type, 4 -type, 2 $d$-type and $1 f$-type STOs centered on the C atom and, $3 s$-type and $1 p$-type STOs centered on the $\mathrm{H}$ atom. These STOs were used to build a basis of 28 molecular orbitals comprising of $16 \sigma, 8 \pi, 3 \delta$ and $1 \phi$ orbitals. These were then used in an initial SCF calculation on the $\mathrm{X}^{1} \Sigma^{+}$state of $\mathrm{CH}^{+}$. Finally, $16 \sigma, 8 \pi$ and $3 \delta$ SCF orbitals were used in a complete active space (CAS) configuration interaction (CI) calculation.

We have tested different target models, a summary of which is presented in Table 1. Finally, target model 5 with an extended $(1 \sigma)^{2}(2-8 \sigma, 1-3 \pi, 1 \delta)^{4}$ CAS was chosen as it gave the best agreement in terms of excitation energies and dipole moments with the coupled cluster singles doubles and triples (CCSDT) calculations of Kowalski \& Piecuch (2001) and the multi reference configuration interaction (MRCI) calculation of Biglari et al (2014). Figure 1 shows the behaviour of the lowest $9 \mathrm{CH}^{+}$target potential energy curves (PECs). Shown also are the corresponding PECs obtained by Biglari et al (2014). In Figure 1 we have shifted the $R$-matrix curves down by $1.28 \mathrm{eV}$ so that the two sets of curves have the same minima of the $\mathrm{X}^{1} \Sigma^{+}$ground state. We find general good agreement between the two sets of curves, though there are some deviations in the higher lying curves of $\mathrm{b}^{1} \Delta, \mathrm{c}^{1} \Sigma^{+}$and the $\mathrm{d}^{1} \Pi$ symmetries.

\subsection{The scattering model}

Our scattering calculations used $12 \mathrm{CH}^{+}$SCF orbitals and a target CAS defined in Section 2.2. These were supplemented by continuum orbitals $F_{i j}$ needed to describe the scattering electron. These were obtained as a truncated partial wave expansion around the centre of mass and partial waves with $l \leq 6$ and $m \leq 2$ were retained in the calculation as these range of values of $l$ and $m$ gave converged results with respect 
Table 1. Comparison of the ground state energy (in Hartree), and excitation energies (in $\mathrm{eV}$ ) from the ground states to 9 low lying states of $\mathrm{CH}^{+}$for different target models. Shown also are a comparison of the ground state dipole moment (in au). Absolute values of our dipole moments are presented since they differ in sign from those of Biglari et al (2014) due to differences in convention. The target models used are the following:

Model 1: $(1-6 \sigma, 1-2 \pi)^{6}$

Model 2: $(1 \sigma)^{2}(2-6 \sigma, 1-2 \pi)^{4}$

Model 3: $(1 \sigma)^{2}(2-8 \sigma, 1-2 \pi, 1 \delta)^{4}$

Model 4: $(1 \sigma)^{2}(2-6 \sigma, 1-3 \pi)^{6}$

Model 5: $(1 \sigma)^{2}(2-8 \sigma, 1-3 \pi, 1 \delta)^{4}$

Model 6: $(1 \sigma)^{2}(2-10 \sigma, 1-3 \pi, 1 \delta)^{4}$.

\begin{tabular}{lrrrrrrr}
\hline Target state & Model 1 & Model 2 & Model 3 & Model 4 & Model 5 & Model 6 & Theory \\
\hline $\mathrm{X}^{1} \Sigma^{+}$ & -37.9626 & -37.9623 & -37.9755 & -37.9689 & -37.9816 & -37.9912 & $-38.0196^{a}$ \\
$\mathrm{~B}^{1} \Sigma^{+}$ & 8.79 & 8.79 & 8.84 & 8.52 & 8.57 & 8.70 & $8.549^{a}$ \\
$\mathrm{C}^{1} \Sigma^{+}$ & 15.03 & 15.03 & 14.75 & 14.91 & 14.63 & 14.61 & $13.525^{a}$ \\
$\mathrm{~A}{ }^{1} \Pi$ & 3.67 & 3.67 & 3.45 & 3.49 & 3.25 & 3.23 & $3.23^{a}$ \\
$\mathrm{~B}^{1} \Pi$ & 14.66 & 14.66 & 14.17 & 14.60 & 14.10 & 14.15 & $14.127^{a}$ \\
$\mathrm{~A}^{1} \Delta$ & 7.50 & 7.50 & 7.35 & 7.27 & 7.14 & 7.32 & $6.964^{a}$ \\
$\mathrm{~B}^{1} \Delta$ & 17.88 & 17.88 & 17.08 & 17.40 & 16.64 & 16.71 & $16.833^{a}$ \\
$\mathrm{a}^{3} \Pi$ & 1.30 & 1.29 & 1.25 & 1.19 & 1.14 & 1.21 & $1.16496^{b}$ \\
$\mathrm{~b}^{3} \Sigma^{-}$ & 5.18 & 5.18 & 5.13 & 4.96 & 4.89 & 5.05 & $4.64268^{b}$ \\
\hline Dipole moment & 0.6227 & 0.7051 & 0.6860 & 0.6867 & 0.6308 & 0.6812 & $0.6501^{a}$ \\
\hline
\end{tabular}

${ }^{a}$ Kowalski \& Piecuch (2001)

${ }^{b}$ Biglari et al (2014)

to bound state energies and resonance positions. The radial Coulomb functions were generated as numerical solutions of an isotropic Coulomb potential and solutions with an energy below 10 Ryd were retained. An $R$-matrix radius of $11 \mathrm{a}_{0}$ was used and this produced $175(70 \sigma, 58 \pi, 47 \delta)$ continuum functions which were Schmidt orthogonalised to the target SCF orbitals. Scattering calculations were then performed using the $(1 \sigma)^{2}(2-8 \sigma, 1-3 \pi, 1 \delta)^{4}$ CAS-CI target wave function for a single geometry, namely the $\mathrm{CH}^{+}$equilibrium geometry $R_{e}=2.137 \mathrm{a}_{0}$.

Extensive tests were made with different scattering models by varying the number and nature of the target states used. Our final scattering models for the different $\mathrm{CH}$ states, and the number and nature of the targets states used are shown in Table 2. These were chosen so as to give the lowest energy of $\mathrm{X}^{2} \Pi$ ground state of $\mathrm{CH}$ and the best vertical excitation energies compared to existing works of van Dishoeck (1987), Song et al (2008) and experiments where available . 


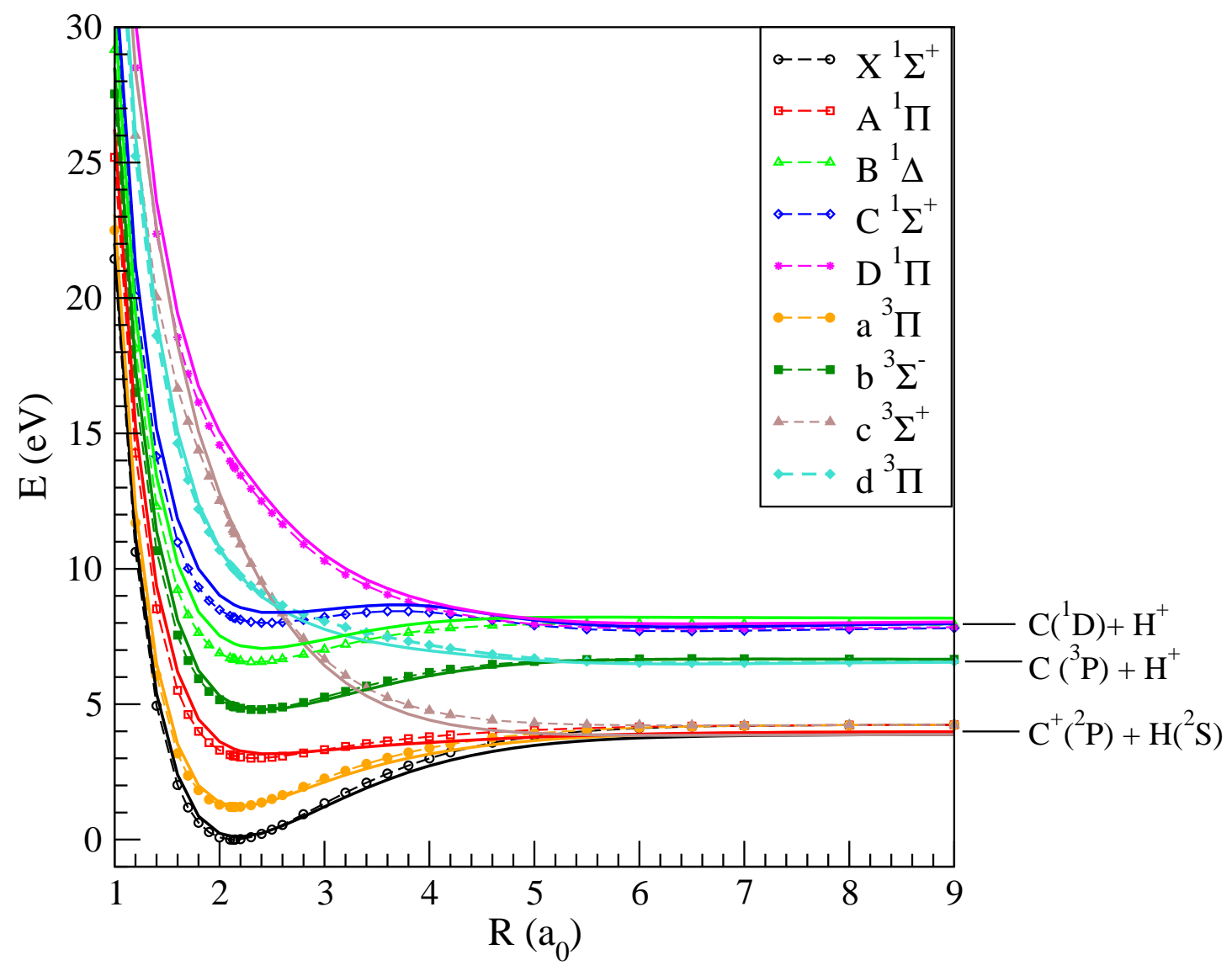

Figure 1. Potential energy curves of the $\mathrm{CH}^{+}$target states used in the calculation. Continuous curves: Present calculation shifted down by $1.28 \mathrm{eV}$. Dashed curves with symbols: Biglari et al (2014).

Table 2. Symmetry and number of states used in the close coupling equation 4 . The target states of lowest energy were used in each case.

Symmetry Number Target states coupled

$\begin{array}{ccl}{ }^{2} \Pi & 8 & \text { two }{ }^{1} \Sigma^{+} \text {, one each of }{ }^{1} \Pi,{ }^{1} \Delta,{ }^{3} \Sigma^{+}{ }^{3} \Sigma^{-} \text {and two }{ }^{3} \Pi \\ { }^{2} \Sigma^{+} & 7 & \text { two }{ }^{1} \Sigma^{+} \text {, one each of }{ }^{1} \Pi,{ }^{1} \Delta,{ }^{3} \Sigma^{+} \text {and two }{ }^{3} \Pi \\ { }^{2} \Sigma^{-} & 5 & \text { one each of }{ }^{1} \Sigma^{-},{ }^{1} \Pi,{ }^{1} \Delta \text { and two }{ }^{3} \Pi \\ { }^{2} \Delta & 7 & \text { one each of }{ }^{1} \Sigma^{+},{ }^{1} \Pi,{ }^{1} \Delta,{ }^{3} \Sigma^{+},{ }^{3} \Sigma^{-} \text {and two }{ }^{3} \Pi \\ { }^{4} \Pi & 5 & \text { one each of }{ }^{3} \Sigma^{+},{ }^{3} \Sigma^{-},{ }^{3} \Delta \text { and two }{ }^{3} \Pi \\ { }^{4} \Sigma^{+} & 3 & \text { one }{ }^{3} \Sigma^{-} \text {and two }{ }^{3} \Pi \\ { }^{4} \Delta & 4 & \text { one each of }{ }^{3} \Sigma^{+},{ }^{3} \Sigma^{-} \text {and two }{ }^{3} \Pi\end{array}$




\section{Results}

In this section we report our calculation for the bound states of $\mathrm{CH}$, cross sections for electronic excitation from the $\mathrm{CH}^{+} \mathrm{X}^{1} \Sigma^{+}$ground state and electron impact dissociation at the $\mathrm{CH}^{+}$equilibrium geometry $R_{e}=2.137 \mathrm{a}_{0}$.

\subsection{Bound states}

An $R$-matrix was constructed at the boundary using the inner region solutions obtained as outlined above. In the outer region, the potential was given by the diagonal and off-diagonal dipole and quadrupole moments of the $\mathrm{CH}^{+}$target states in addition to the Coulomb potential. The outer region bound wave functions were propagated in this potential to $40 \mathrm{a}_{0}$ using the improved Runge-Kutta-Nystrom procedure implemented by Zhang et al (2011) to the asymptotic region, where they were matched with asymptotic solutions obtained from a Gailitis expansion (Noble \& Nesbet 1984). Bound states were then found using the searching algorithm of Sarpal et al (1991) with the improved nonlinear, quantum defect based grid of Rabadán \& Tennyson (1996). These calculations were performed at the $\mathrm{CH}$ equilibrium geometry $R_{e}=2.116 \mathrm{a}_{0}$ as this facilitates direct comparison with other studies.

Table 3 shows the vertical excitation energies from the $\mathrm{CH} \times{ }^{2} \Pi$ ground state. Shown also are the results from van Dishoeck (1987), Song et al (2008) and the experimental values given by Herzberg \& Johns (1969). Our excitations energies are within $0.2 \mathrm{eV}$ of the corresponding energies of other reported calculations presented in the table.

The ionization potential (IP) of $\mathrm{CH}$ has been reported by some authors. For the $\mathrm{X}^{2} \Pi$ ground state, Tennyson (1988) reported an ionization potential of $10.83 \mathrm{eV}$ which agreed reasonably with the $10.64 \mathrm{eV}$ measurement of Herzberg \& Johns (1969). Our estimate for the ionization potential at $R_{e}=2.116 \mathrm{a}_{0}$ is about $10.43 \mathrm{eV}$ and is $2 \%$ from the experimental value of Herzberg \& Johns (1969).

\subsection{Resonances}

It is well known that in an adiabatic fixed nuclei calculation, diabatic neutral states lying in the continuum above the ion appear as resonances having finite widths that are inversely proportional to their lifetimes. These states play an important role in several collision processes such as vibrational excitation and dissociative recombination. Here we try to estimate the position and widths of the resonant states at a single geometry, $R_{e}=2.137 \mathrm{a}_{0}$.

For calculation of resonances, the $R$-matrix were propagated (Morgan 1984) to $70 \mathrm{a}_{0}$, as tests showed that this produced stable results. It was then matched with asymptotic Coulomb functions (Barnett 1982) obtained using the Gailitis expansion procedure of Noble \& Nesbet (1984). Resonances were detected and fitted to a BreitWigner profile to obtain their energy $(\mathrm{E})$ and width $(\Gamma)$ using the RESON program 
Table 3. Vertical excitation energies (in $\mathrm{eV}$ ) from the $\mathrm{X}{ }^{2} \Pi$ ground states of the $\mathrm{CH}$ molecule at $\mathrm{CH}$ equilibrium bond lenght $R=2.116 \mathrm{a}_{0}$.

\begin{tabular}{|c|c|c|c|c|}
\hline $\mathrm{CH}$ state & This work & van Dishoeck ${ }^{a}$ & Song et $a l^{b}$ & Experiment $^{c}$ \\
\hline $\mathrm{X}^{2} \Pi$ & 0.0 & 0.0 & 0.0 & 0.0 \\
\hline $\mathrm{a}^{4} \Sigma^{-}$ & 0.60 & 0.71 & 0.67 & 0.74 \\
\hline $\mathrm{A}^{2} \Delta$ & 3.04 & 3.00 & 2.91 & 2.88 \\
\hline $\mathrm{B}^{2} \Sigma^{-}$ & 3.33 & 3.24 & 3.17 & 3.19 \\
\hline $\mathrm{C}^{2} \Sigma^{+}$ & 4.06 & 4.02 & 3.89 & 3.94 \\
\hline $2^{2} \Sigma^{+}$ & 6.42 & 6.39 & & \\
\hline $2^{2} \Pi$ & 7.52 & 7.43 & & 7.31 \\
\hline $3^{2} \Pi$ & $7.72^{*}$ & 7.94 & & 7.96 \\
\hline $3^{2} \Sigma^{+}$ & 7.92 & 7.96 & & 8.00 \\
\hline $1^{4} \Pi$ & 7.50 & 7.55 & & \\
\hline $4^{2} \Sigma^{+}$ & 8.59 & 8.63 & & \\
\hline $2^{2} \Delta$ & 8.87 & 8.93 & & 9.05 \\
\hline $4^{2} \Pi$ & 8.87 & 8.05 & & \\
\hline $2{ }^{4} \Pi$ & 8.83 & 8.90 & & \\
\hline $2^{2} \Sigma^{-}$ & 9.04 & 9.06 & & \\
\hline $1^{4} \Delta$ & 9.05 & 9.10 & & \\
\hline $5^{2} \Pi$ & 9.13 & 9.04 & & \\
\hline $3^{2} \Delta$ & 9.36 & 9.39 & & \\
\hline \multicolumn{5}{|c|}{${ }^{a}$ van Dishoeck (1987) } \\
\hline \multicolumn{5}{|c|}{${ }^{b}$ Song et al (2008) } \\
\hline${ }^{c}$ Herzberg & Johns $(19$ & & & \\
\hline
\end{tabular}

(Tennyson \& Noble 1984) with an energy grid $0.5 \times 10^{-3}$ Ryd. The magnitudes of the complex quantum defects $\mu=\alpha+i \beta$ were obtained using the relations (Tennyson 1988)

$$
E_{r}=E_{t}-\frac{1}{\nu^{2}}, \quad \Gamma=\frac{2 \beta}{\nu^{3}}
$$

where the effective quantum number $\nu$ equals $n-\alpha$ and $E_{t}$ is the energy of the threshold to which the Rydberg series converges.

Tables 4 and 5 shows some of the resonances, their widths and effective quantum numbers for $\mathrm{CH}$ at $R_{e}=2.137 \mathrm{a}_{0}$ for doublet and quartet states, respectively. The resonances are presented in terms their $\mathrm{CH}^{+}$parent electronic state. The effective quantum numbers are calculated assuming the resonances can be associated with this parent state. Many of the resonances in the tables are seen to form a series with respect to their effective quantum numbers. The behaviour of these resonance series and their widths are the starting point for the construction of dissociative states and electronic couplings that are the basis for dissociative recombination (Little et al 2014, Mezei et al 2016, Laporta et al 2017). 
Table 4. Resonance positions and widths (in Ryd) and effective quantum numbers at $R=2.137 \mathrm{a}_{0}$ for some doublet states of the $e^{-}-\mathrm{CH}^{+}$system below the first two $\mathrm{CH}^{+}$ excited states. Numbers within brackets indicate power of 10 .

\begin{tabular}{cccccc}
\hline \multicolumn{3}{c}{ Below a ${ }^{3} \Pi$ state } & \multicolumn{3}{c}{ Below A ${ }^{1} \Pi$ state } \\
Position & Width & $\nu$ & Position & Width & $\nu$ \\
\hline${ }^{2} \Pi$ & & & & & \\
0.0134 & $0.6872(-03)$ & 3.7743 & 0.0933 & $0.1333(-01)$ & 2.6222 \\
0.0199 & $0.3979(-04)$ & 3.9639 & 0.1281 & $0.5984(-03)$ & 3.0060 \\
0.0317 & $0.1137(-02)$ & 4.3889 & 0.1481 & $0.3994(-02)$ & 3.3207 \\
0.0383 & $0.1585(-03)$ & 4.6973 & 0.1606 & $0.4663(-02)$ & 3.5770 \\
0.0430 & $0.2552(-04)$ & 4.9626 & 0.1739 & $0.1945(-03)$ & 3.9247 \\
0.0464 & $0.4230(-03)$ & 5.1885 & 0.1854 & $0.1790(-02)$ & 4.3279 \\
0.0514 & $0.1004(-03)$ & 5.5727 & 0.1908 & $0.2025(-02)$ & 4.5646 \\
0.0600 & $0.2141(-03)$ & 6.5166 & 0.1984 & $0.1376(-03)$ & 4.9755 \\
0.0619 & $0.2913(-03)$ & 6.7941 & 0.2019 & $0.6828(-03)$ & 5.2058 \\
$2 \Sigma^{-}$ & & & & & \\
0.0370 & $0.5528(-03)$ & 2.9086 & 0.3324 & $0.6312(-03)$ & 3.0324 \\
0.0675 & $0.6537(-03)$ & 3.3773 & 0.3796 & $0.1938(-03)$ & 4.0312 \\
0.0894 & $0.2046(-03)$ & 3.8998 & 0.4015 & $0.1053(-03)$ & 5.0267 \\
0.1030 & $0.1655(-03)$ & 4.3774 & 0.4136 & $0.6336(-04)$ & 6.0237 \\
0.1134 & $0.7511(-04)$ & 4.8935 & & & \\
0.1206 & $0.5528(-04)$ & 5.3753 & & & \\
0.1306 & $0.2767(-04)$ & 6.3729 & & & \\
0.1341 & $0.1847(-04)$ & 6.8853 & & & \\
$2 \Delta$ & & & & & \\
0.0319 & $0.2427(-04)$ & 4.4005 & 0.1044 & $0.1741(-01)$ & 2.7280 \\
0.0496 & $0.1137(-03)$ & 5.4221 & 0.1382 & $0.2415(-01)$ & 3.1527 \\
0.0595 & $0.7277(-03)$ & 6.4447 & 0.1465 & $0.2844(-04)$ & 3.2913 \\
0.0657 & $0.1682(-03)$ & 7.4813 & 0.1666 & $0.5492(-05)$ & 3.7212 \\
\hline & & & & &
\end{tabular}

\subsection{Electron impact excitation}

Figure 2 shows the vibrationally-resolved electronic-excitation cross sections from the $\mathrm{X}{ }^{1} \Sigma^{+}$vibrational ground states to the $\mathrm{a}^{3} \Pi, \mathrm{A}{ }^{1} \Pi$ and $\mathrm{b}^{3} \Sigma^{-}$excited states, the vibrational state $\nu^{\prime}$ being indicated in each panel. To calculate the vibrationally resolved cross sections we first calculated Franck-Condon factors corresponding to the transitions from the a ${ }^{3} \Pi, \mathrm{A}^{1} \Pi$ and $\mathrm{b}^{3} \Sigma^{-}$states to the $\mathrm{X}^{1} \Sigma^{+}$state with the potential energy curves of Biglari et al (2014) using Le Roy's LEVEL program (Le Roy 2017), and these are presented in Table 6 . At least for the $\mathrm{A}{ }^{1} \Pi \rightarrow X^{1} \Sigma^{+}$transitions, these are in good agreement to within $10 \%$ of the Franck-Condon factors computed with more accurate potential energy curves (Hakalla et al 2006). We did not use the Franck-Condon factors computed with our CI curves of Figure 1 since these were not as accurate as the Franck- 
Table 5. Resonance positions and widths (in Ryd) and effective quantum numbers at $R=2.137 \mathrm{a}_{0}$ for some quartet states of the $\mathrm{e}-c \mathrm{H}^{+}$system below the first two $\mathrm{CH}^{+}$ excited states. Numbers within brackets indicate power of 10 .

\begin{tabular}{|c|c|c|c|c|c|}
\hline \multicolumn{3}{|c|}{ Below a ${ }^{3} \Pi$ state } & \multicolumn{3}{|c|}{ Below A ${ }^{1} \Pi$ state } \\
\hline Position & Width & $\nu$ & Position & Width & $\nu$ \\
\hline \multicolumn{6}{|l|}{${ }^{4} \Pi$} \\
\hline 0.0980 & $0.3412(-02)$ & 2.3684 & 0.5263 & $0.4887(-01)$ & 2.0909 \\
\hline 0.1598 & $0.7635(-03)$ & 2.9298 & 0.5917 & $0.7974(-02)$ & 2.4744 \\
\hline 0.1886 & $0.5668(-03)$ & 3.3765 & 0.6399 & $0.1414(-02)$ & 2.9467 \\
\hline 0.2111 & $0.3134(-03)$ & 3.9170 & 0.6704 & $0.2675(-02)$ & 3.4371 \\
\hline 0.2242 & $0.2237(-03)$ & 4.3805 & 0.6900 & $0.5549(-03)$ & 3.9192 \\
\hline 0.2348 & $0.1597(-03)$ & 4.9118 & 0.7036 & $0.8360(-03)$ & 4.4093 \\
\hline 0.2417 & $0.1132(-03)$ & 5.3825 & 0.7135 & $0.2518(-03)$ & 4.9069 \\
\hline $\begin{array}{c}0.2476 \\
{ }^{4} \Sigma^{-}\end{array}$ & $0.9161(-04)$ & 5.9091 & 0.7207 & $0.3149(-03)$ & 5.3900 \\
\hline 0.0840 & $0.6302(-02)$ & 2.2806 & & & \\
\hline 0.1363 & $0.5288(-02)$ & 2.6726 & & & \\
\hline 0.1600 & $0.5792(-03)$ & 2.9325 & & & \\
\hline 0.1848 & $0.1741(-02)$ & 3.3074 & & & \\
\hline 0.1999 & $0.1882(-02)$ & 3.6195 & & & \\
\hline 0.2118 & $0.2072(-03)$ & 3.9399 & & & \\
\hline 0.2225 & $0.7533(-03)$ & 4.3142 & & & \\
\hline $\begin{array}{l}0.2291 \\
{ }^{4} \Delta\end{array}$ & $0.8264(-03)$ & 4.6022 & & & \\
\hline 0.1665 & $0.7112(-04)$ & 3.0178 & 0.5747 & $0.6877(-02)$ & 2.3543 \\
\hline \multirow[t]{3}{*}{0.2137} & $0.5385(-04)$ & 3.9987 & 0.6409 & $0.7390(-03)$ & 2.9595 \\
\hline & & & 0.6907 & $0.2092(-03)$ & 3.9426 \\
\hline & & & 0.7267 & $0.5190(-04)$ & 5.9343 \\
\hline
\end{tabular}

Condon factors of Biglari et al (2014) for the $\mathrm{A}^{1} \Pi \rightarrow X^{1} \Sigma^{+}$transitions.

To get the vibrationally resolved cross sections, subsequently the cross sections were scaled with the Franck-Condon factor corresponding to the $\Gamma\left(\nu^{\prime}=j\right) \rightarrow X^{1} \Sigma^{+}\left(\nu^{\prime \prime}=0\right)$ transitions, where $\Gamma$ is one of the excited states mentioned above and $j=0,1,2$ or 3 .

The cross sections show numerous oscillations due to the resonance structure associated with excited Rydberg states of $\mathrm{CH}$. A full treatment of the vibrational motion would tend to smooth these out. Noteworthy is also the $\mathrm{X}^{1} \Sigma^{+}\left(\nu^{\prime \prime}=0\right) \rightarrow \mathrm{a}^{3} \Pi\left(\nu^{\prime}=0\right)$ cross section, which is $2-3$ orders of magnitude higher than all others indicating that in the energy range considered almost all the electronic transitions are to the a ${ }^{3} \Pi\left(\nu^{\prime}=0\right)$ state. 
Table 6. Franck-Condon factors used to get the vibrationally resolved cross sections in Figure 2 using the curves of Biglari et al (2014). $\nu^{\prime}$ and $\nu^{\prime \prime}$ label the vibrational levels of the upper states and the $\mathrm{X}^{1} \Sigma^{+}$ground state respectively. The quantities in the brackets are the corresponding Franck-Condon factors of Hakalla et al (2006).

\begin{tabular}{lllll} 
& $\nu^{\prime}=0$ & $\nu^{\prime}=1$ & $\nu^{\prime}=2$ & $\nu^{\prime}=3$ \\
\hline $\mathrm{A}^{1} \Pi-X^{1} \Sigma^{+}$ & & & & \\
$\nu^{\prime \prime}=0$ & $\begin{array}{l}0.6066 \\
(0.6343)\end{array}$ & $\begin{array}{l}0.2644 \\
(0.2580)\end{array}$ & $\begin{array}{l}0.0845 \\
(0.0769)\end{array}$ & $\begin{array}{l}0.0259 \\
(0.0217)\end{array}$ \\
\hline $\mathrm{a}^{3} \Pi-X^{1} \Sigma^{+}$ & & & & \\
$\nu^{\prime \prime}=0$ & 0.9968 & 0.0031 & 0.0001 & 0.0 \\
\hline $\mathrm{b}^{3} \Sigma^{-}-X^{1} \Sigma^{+}$ & & & & \\
$\nu^{\prime \prime}=0$ & 0.6205 & 0.2690 & 0.0808 & 0.0217 \\
\hline
\end{tabular}
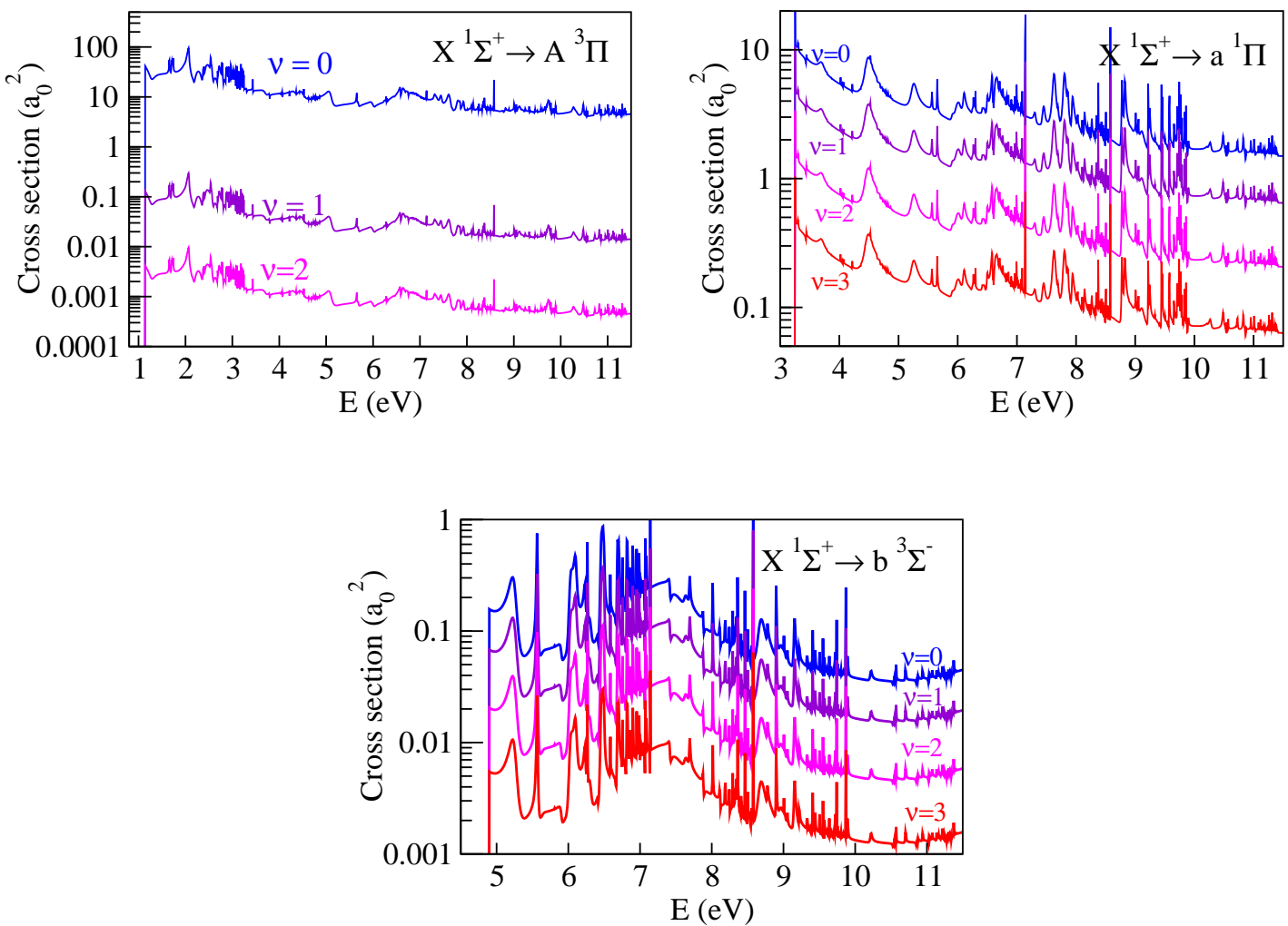

Figure 2. Excitation cross sections from the $\mathrm{X}^{1} \Sigma^{+}$ground state of the $\mathrm{CH}^{+}$molecule to the excited states with vibrational quantum number $\nu^{\prime}$ as indicated in each panel at $R=2.137 \quad \mathrm{a}_{0}$.

\subsection{Electron impact dissociation}

To the best of our knowledge, no theoretical calculation of the electron impact dissociation of $\mathrm{CH}^{+}$have ever been reported. Figure 1 shows the dissociation limits 


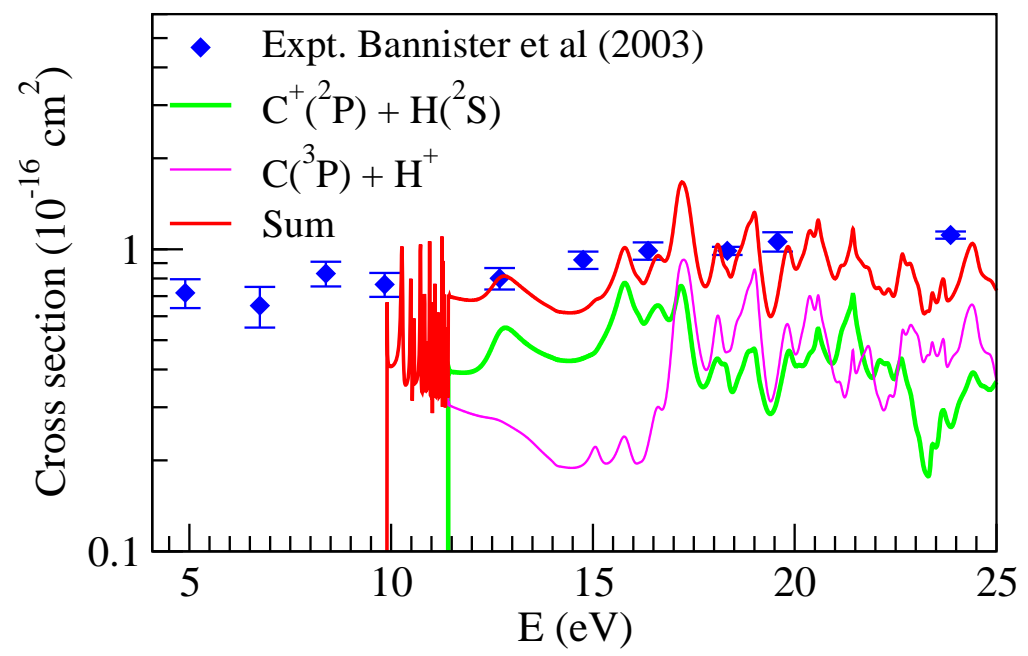

Figure 3. Electron impact dissociation of the $\mathrm{CH}^{+}$molecule Curves as indicated in the panel. Experiment: Bannister et al (2003).

for some the PECs included in this calculation. The $\mathrm{X}^{1} \Sigma^{+}$, a ${ }^{3} \Pi, \mathrm{A}{ }^{1} \Pi$ and $\mathrm{c}^{3} \Sigma^{+}$ states dissociate to the fragments $\mathrm{C}^{+}\left({ }^{2} P\right)+\mathrm{H}\left({ }^{2} S\right)$, while the $\mathrm{b}^{3} \Sigma^{-}$and $\mathrm{d}{ }^{3} \Pi$ states dissociate into the fragments $\mathrm{C}\left({ }^{3} P\right)+\mathrm{H}^{+}$. Experiments measuring the absolute cross sections for electron impact dissociation of $\mathrm{CH}^{+}$ions producing $\mathrm{C}^{+}$ions were reported by Bannister et al (2003). Figure 3 shows the results of Bannister et al (2003) together with our theoretical results for the dissociation to the asymptotic states $\mathrm{C}^{+}\left({ }^{2} P\right)+\mathrm{H}\left({ }^{2} S\right)$ (thick curve), $\mathrm{C}\left({ }^{3} P\right)+\mathrm{H}^{+}$(thin curve) and their sum (topmost curve). Our results were calculated with the assumption that all excitations from the $\mathrm{X}^{1} \Sigma^{+}$ground state to the states, dissociating to a particular asymptote, above the corresponding dissociation threshold result in dissociation. Even at these energies, however, such excitation cross sections would include both dissociation and electronic excitation cross sections, as electronic excitation to the core excited bound states competes with dissociation. The contribution of cross sections corresponding to 'pure' electronic excitation were estimated using Franck-Condon factors and these were then subtracted out to get the dissociation cross sections.

Our results agree reasonably well with the measurements of Bannister et al (2003) from the threshold $(10 \mathrm{eV})$ to about $18 \mathrm{eV}$ and are within $20 \%$ of the measured values. Above this we would expect our results underestimate the cross section due to neglect of higher lying states which were not included in our calculations. However, the appearance of agreement of the summed cross section (top most curve in Figure 3) may be largely coincidental. The experiments were performed on a hot sample of $\mathrm{CH}^{+}$whose state distribution was not determined. This leads to significant measured cross sections from below threshold to dissociation for the $\mathrm{X}^{1} \Sigma^{+}(\nu=0)$ ground state. This may well be due to the presence of a significant population of $\mathrm{CH}^{+}$ions in their a ${ }^{3} \Pi$ metastable state (Bannister et al 2003). Without knowledge of the experimental state distribution 
it is not possible to make a proper comparison with our results.

\section{Conclusions}

In this paper we report $R$-matrix calculations on electron collision with $\mathrm{CH}^{+}$. An initial CI calculation was done for the $\mathrm{CH}^{+}$target states, which were then used in a scattering calculation to yield bound and resonant states of $\mathrm{CH}$ at a single geometry. The $\mathrm{CH}$ bound states agreed well with earlier calculations. We have also calculated cross sections for vibrationally resolved electron impact excitation, and electron impact dissociation of $\mathrm{CH}^{+}$ions. Our electron impact dissociation cross sections are in reasonable agreement with the only available experimental measurement and do not deviate from the experimental values by more than 20\%. However, given the experimental conditions it is not clear if this represents a like-for-like comparison. To the best of our knowledge, this is the only available $a b$ initio theoretical calculation of the dissociation process.

\section{Supplementary data}

The vibrationally resolved electronic excitation and dissociation cross sections presented in this work are available in electronic form as supplementary data.

\section{Acknowledgements}

We are deeply indebted to Robert Le Roy for helping us with his LEVEL16 program used to calculate the Franck-Condon factors used in this work. RG gratefully acknowledges CSIR, New Delhi, for a Junior Research Fellowship. We also thank Ioan Schneider for numerous discussions on the $\mathrm{CH}^{+} / \mathrm{CH}$ states.

\section{References}

Amitay Z, Zajfman D, Forck P, Hechtfischer U, Seidel B, Grieser M, Habs D, Repnow R, Schwalm D and Wolf A 1996 Phys. Rev. A 54, 4032

Bannister M E Krause H F, Vane C R, Djurić N, Popović D B, Stepanović M, Dunn G H, Chung Y S, Smith A C H and Wallbank B 2003 Phys. Rev. A 68, 042714

Barnett A R 1982 Computer Phys. Comms. 27, 147-166

Barinvos G and van Hemert M C 2004 Chem. Phys. Lett. 399, 406-411

Biglari Z, Shayesteh A, Maghari A 2014 Comput. Theoret. Chem. 1047, 22-29

Boukaline F, Grozdanov T P, Andric L and McCaroll R 2005 J. Chem. Phys. 122, 044108

Burke P G 2011 R-Matrix Theory of Atomic Collisions (Springer, Heidelberg) .

Buttle P J A 1967 Phys. Rev. 160, 719-29

Cade P E and Huo W M, 1967 J. Chem. Phys. 47, 614-648

Carata L, Orel A E, Raoult M, Schneider I F and Suzor-Weiner A 2000 Phys. Rev. A 62, 052711

Chakrabarti K \& Tennyson J 2012 Eur. Phys. J. D 66, 31

Cho Y S \& Le Roy R J 2016 J. Chem. Phys. 144, 024311

Douglas A E and Herzberg G 1941 Astrophys. J. 94, 381 
Douglas A E and Herzberg G 1941 Canadian. J. Res. 20, Sec A, 71

Faure A, Halvick P, Stoecklin T, Honvault P, Epee Epee M D, Mezei J Zs, Motapon O, Schneider I F, Tennyson J, Roncero O, Bulut N and Zanche A 2017 Mon. Not. R. astr. Soc. in press.

Godard B, Falgarone E, Gerin M, Lis D C, De Luca M, Black J H, Goicoechea J R, Cernicharo J, Neufeld D A, Menten K M and Emprechtinger M 2012 Astron. Astrophys. 540, A87

Godard B and Cernicharo J 2013 Astron. Astrophys. 550, A8

Hakalla R, Kepa R, Szajna W and Zachwieja M 2006 Eur. Phys. J. D 38,481

Hamilton J R, Faure A and, Tennyson J 2016 Mon. Not. R. Astron. Soc. 455, 3281

Hayden Smith Wm, Liszt H S and Lutz B L 1973 Astrophys. J. 183, 69-80

Herzberg G and, Johns J W C 1969 Astrophysics J. 158,399

Kanzler A W Sun H and Freed K F 1991 Int. J. Quant. Chem. XXXIX, 269-286

Kowalski K and Piecuch P 2001 Chem. Phys. Lett. 347, 237-246

Laporta V, Chakrabarti K, Celiberto R, Janev R K, Mezei J Zs, Niyonzima S, Tennyson J \& Schneider I F 2017 Plasma Phys. Control. Fusion, 59, 045008

Le Roy R J 2017 J. Quant. Spectrosc. Radiat. Transf. 186, 167 - 178.

Lim A J, Rabadán I and Tennyson J 1999 Mon. Not. R. Astron. Soc. 306, 473-478

Liszt H S \& Hayden Smith Wm 1972 J. Quant. Spectrosc. Radiat. Transfer., 412, 947

Little D A, Chakrabarti K, Mezei J Zs, Schneider I F \& Tennyson J 2014 Phys. Rev. A, 90, 052705

Lorquet A J, Lorquet J C, Wankenne H, Momigny J \& Lefebvre-Brion H 1971 J. Chem. Phys, 55, 4053

Mezei J Zs, Colboc F, Pop N, Ilie S, Chakrabarti K, Niyonzima S, Lepers M, Bultel A, Dulieu O, Motapon O, Tennyson J,Hassouni K and Schneider I F 2016 Plasma Sources Sci. Technol. 25, 055022

Morgan L A 1984 Comput. Phys. Commun. 31, 419.

Morgan L A, Tennyson J \& Gillan C J 1998 Computer Phys. Commun. 114, 120-128.

Myers A T, McKee C F, Li P S 2015 Mon. Not. R. Astron. Soc. 453, 2747

Nagy Z, Van der Tak F F S, Ossenkopf V, Gerin M, Le Petit F, Le Bourlot J, Black J H, Goicoechea

J R, Joblin C, Rllig M and Bergin E A 2013 Astron. Astrophys. 550, A96

Noble C J \& Nesbet R K 1984 Computer Phys. Comms. 33, 399-411

Rabadán I \& Tennyson J 1996 J. Phys. B: At. Mol. Opt. Phys. 29, 3747-3761

Rabadán I \& Tennyson J 1997 J. Phys. B: At. Mol. Opt. Phys. 30, 1975-1988

Sarpal B K, Branchett S E, Tennyson J \& Morgan L A 1991 J. Phys. B: At. Mol. Opt. Phys. 24, 36853699.

Sauer S P A \& S̆pirko 2013 J. Chem. Phys. 138, 024315

Song C, Han H, Zhang Y, Yu Y and Gao T 2008 Can. J. Phys. 86, 1145-1151

Tawara H, in Atomic and Molecular Processes in Fusion Plasmas, edited by R. K. Janev Plenum, New York, 1995, pp. 461-496.

Tennyson J 1988 J. Phys. B: At. Mol. Opt. Phys., 21,'805-816.

Tennyson J \& Morgan L A 1999 Phil. Trans. Royal Soc. London A 357, 1161-1173

Tennyson J and Noble C J 1984 Comput. Phys. Commun. 33, 421-424

Tennyson J 1988 J. Phys. B: At. Mol. Opt. Phys. 21, 805-816

Tennyson J 2010 Phys. Rep. 491, 29-76.

van Dishoeck E F 1987 J. Chem. Phys. 86, 196-214

Zhang R, Baluja K L, Franz J \& Tennyson J 2011 J. Phys. B: At. Mol. Opt. Phys. 44, 035203 